\title{
A Multi-Wavelength View of the XMM-Newton Galactic Plane
}

\author{
Ada Nebot Gómez-Morán ${ }^{1}$, Christian Motch ${ }^{1}$, on behalf of the XMM-Newton Survey Science \\ Centre \\ ${ }^{1}$ Observatoire Astronomique de Strasbourg, Université de Strasbourg, CNRS, UMR 7550, 11 rue de l'Université, 67000 \\ Strasbourg, France.
}

Corresponding author: ada.nebot@astro.unistra.fr

\begin{abstract}
We present an X-ray survey of the Galactic Plane conducted by the Survey Science Centre of the XMM-Newton satellite. The survey contains more than 1300 X-ray detections at low and intermediate Galactic latitudes and covering 4 deg ${ }^{2}$ well spread in Galactic longitude. From a multi-wavelength analysis, using optical spectra and helped by optical and infrared photometry we identify and classify about a fourth of the sources. The observed surface density of soft X-ray $(<2 \mathrm{keV})$ sources decreases with Galactic latitude and although compatible with model predictions at first glance, presents an excess of stars, likely due to giants in binary systems. In the hard band $(>2 \mathrm{keV})$ the surface density of sources presents an excess with respect to the expected extragalactic contribution. This excess highly concentrates towards the direction of the Galactic Centre and is compatible with previous results from Chandra observations around the Galactic Centre. The nature of these sources is still unknown.
\end{abstract}

Keywords: stars - binaries - spectroscopy - photometry - IR - optical - X-rays.

\section{Introduction}

Galactic X-ray surveys can help us to learn about the Galaxy's structure, stellar formation and evolution. Since long we know that the soft X-ray emission $(<2 \mathrm{keV})$ of the Galaxy is dominated by stars. But the X-ray luminosity function of hard X-ray emitting sources $(>2 \mathrm{keV})$ is still not well constrained, in particular at low to intermediate X-ray luminosities $\left(10<\mathrm{L}_{\mathrm{X}}<10^{34} \mathrm{erg} \mathrm{s}^{-1}\right)$. Cataclysmic variables and active binaries contribute at X-ray luminosities up to $\sim 10^{33} \mathrm{erg} \mathrm{s}^{-1}$ (Sazonov et al. 2006), but recent results show that other sources, such as massive OB stars, Wolf-Rayet and wind colliding binaries also contribute to this $\mathrm{L}_{\mathrm{X}}$ range (Mauerhann et al. 2010, Anderson et al. 2011). A census of these populations is thus needed.

Discrepancies between observations and model predictions exist since the first X-ray surveys of the Galaxy were carried out by the Einstein satellite. Stellar population models predict less stars than we observe, in particular, there is an excess of stars with spectral type $\mathrm{K}$, this is the so called "yellow excess". The origin of this excess is highly debated and there are two possible explanations: either these $\mathrm{K}$ stars are young stars or old evolved giants, which can be either single or in binary systems (Favata et al. 1988, Sciortino et al. 1995).

$\mathrm{X}$-ray surveys can reveal concentrations of specific populations in certain regions of the Galaxy: high-mass X-ray binaries (HMXB) seem to be concentrated in the
Norma Arm (Lutovinov et al. 2005); the Gould Belt contains a young population of late type stars (Guillout et al. 1998). Last but not least, different populations of theoretically predicted X-ray binaries are still to be discovered. Binary population models predict a population of X-ray binaries at low luminosities, preHMXBs and pre-low-mass X-ray binaries that would be accreting matter coming from the wind of the companion star at low rates rather than from Roche lobe overflow (Willems \& Kolb 2003, Pfahl et al 2002). Other predicted objects are HMXBs with white dwarf as compact object instead of a classical HMXB where the compact object is a neutron star (Motch et al 2007). So far, none of these objects is confirmed observationally. To learn about all these questions we need to study the X-ray content of the Galaxy.

\section{XMM-Newton Survey Science Centre}

The XMM-Newton satellite can help us to give answer to these questions. Thanks to it's large field of view and it's high sensitivity, each XMM-Newton pointed observation discovers from 30 to 100 serendipitous sources. The XMM-Newton Survey Science Centre (SSC) generates catalogues of serendipitous sources and publishes them on a regular basis. The latest available catalogue, the 2XMMi-DR3 catalogue, contains about 250000 unique sources, and the 3XMM catalogue will 
soon be released containing more than half a million detections corresponding to about 370000 unique sources. One of the goals of the SSC is to characterise and identify these sources in an statistical manner. For that, specific surveys have been studied in great detail, such as a bright flux sample by Della Ceca et al. 2004, a medium flux sample by Barcons et al. 2002 and the Galactic survey from Hands et al. 2004, with spectro- scopic identification carried out by Motch et al 2010 . These surveys serve as learning samples for an statistical identification. In this paper we briefly describe the main results of a survey carried out by the XMMNewton SSC in the Galactic Plane. For details on the survey we refer the reader to Nebot Gómez-Morán et al. 2013.

\section{WRIIO}
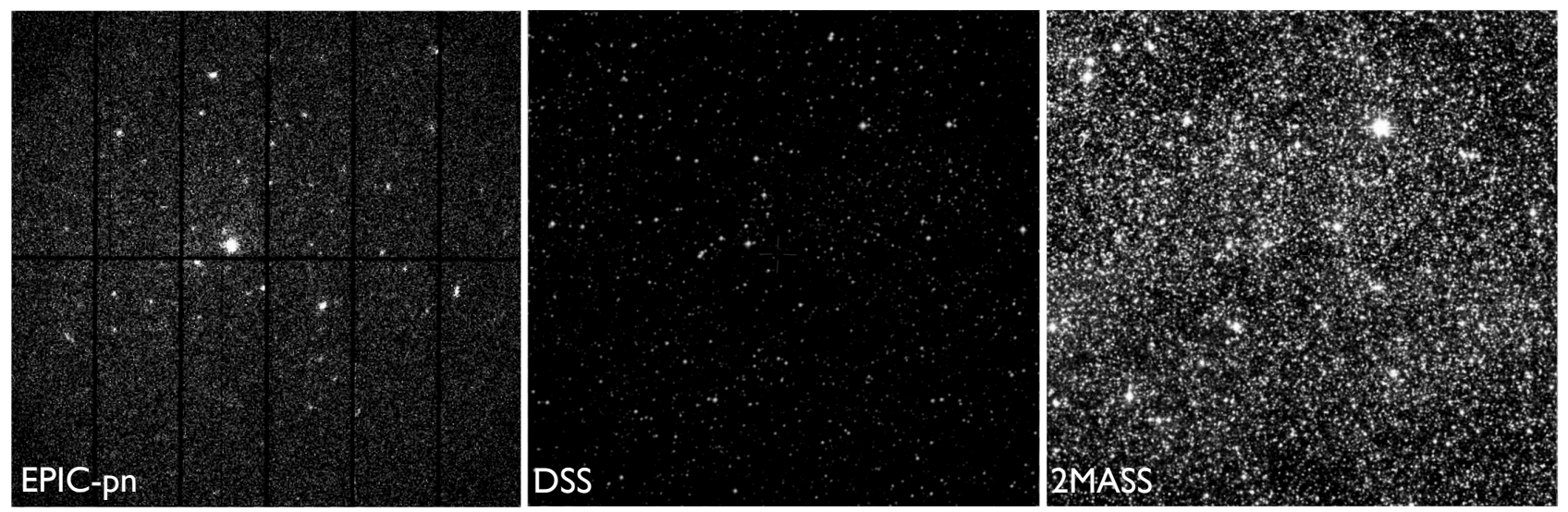

Figure 1: Example field studied in this survey. From left to right, X-ray (EPIC-pn), optical (DSS) and infrared (2MASS) images around the WR 110.

\section{The Galactic Plane Survey}

The XMM-Newton SSC survey of the Galactic Plane contains 26 pointings, covering about $4^{\circ}$ at low and intermediate Galactic latitudes $\left(|b|<20^{\circ}\right)$ and well spread in Galactic longitude. It contains about 1300 detections up to limiting fluxes of $\sim 2 \times$ $10^{-15} \mathrm{erg} \mathrm{cm}^{-2} \mathrm{~s}^{-1}$ in the soft $(\mathrm{kT}<2 \mathrm{keV})$ band and $\sim$ $10^{-14} \mathrm{erg} \mathrm{cm}^{-2} \mathrm{~s}^{-1}$ in the hard band $(2<\mathrm{kT}<12 \mathrm{keV})$.

\subsection{Multi-wavelength identification}

In order to identify these sources in the optical and the infrared we cross-correlated the X-ray positions with large optical and infrared published catalogues, such as the SDSS, USNO and 2MASS. We show an example of one of the fields studied in this survey in Figure 1. The XMM-Newton detected about 50 sources around the pointed observation of WR 110. If we look in the optical (middle panel) the field clearly contains a large number of sources, while in the infrared (right panel) the field becomes very crowded. This example reflects the difficulty of multi-wavelength identification of X-ray sources in the Galactic Plane. Due to the relatively large X-ray positional errors, and the high surface density of sources at faint fluxes, only not too distant and relatively bright sources can be identified in the optical and the infrared.
Among the $\sim 1300 \mathrm{X}$-ray detected sources we could find counterparts in the optical to about $60 \%$, number which drops to about $40 \%$ in the infrared.

\subsection{Source classification}

A spectroscopic campaign was carried out to classify the sources. About one forth of the detected X-ray sources are classified. Most of them are active coronae, but we also found other interesting objects, such as young $\mathrm{T}$ Tauri and Herbig-Ae stars, and old cataclysmic variables. We identify four Be stars with X-ray luminosities around $10^{32} \mathrm{erg} \mathrm{s}^{-1}$ a value too high for normal Be stars. These systems thus belong to the class of $\gamma$-Cas analogues, which means that we have almost doubled the number of $\gamma$-Cas objects known until the date (see talk by C. Motch in this congress).

We cross-matched our X-ray sources with other published catalogues making use of Vizier and the XCat-DB databases, and produced spectral energy distributions which helped us to discern the type of source. Infrared data from the WISE catalogue revealed one of the T Tauri has a transition disk, which highlights the relevance of having a multi-wavelength view of high energy sources. 
A Multi-Wavelength View of the XMM-Newton Galactic Plane

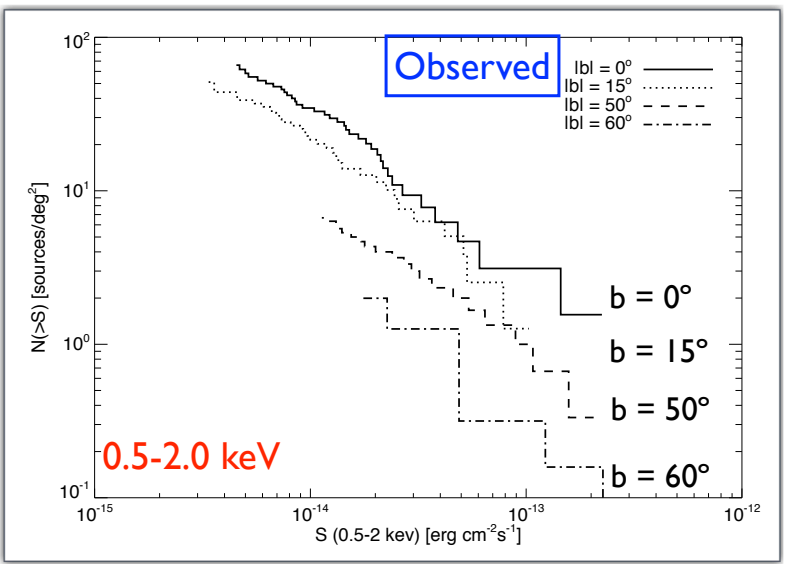

Figure 2: $\log \mathrm{N}-\log \mathrm{S}$ curves in the soft band $(<2 \mathrm{keV})$ and as a function of Galactic latitude. We included the data from Lopez-Santiago et al. 2007 and Barcons et al. 2007 at high Galactic latitudes.

Using infrared colour-colour diagrams for the stellar content of our survey we could distinguish main sequence stars from evolved giants. On the one hand, due to magnetic braking, stellar rotation decreases with the age of the stars. On the other hand the X-ray luminosity is strongly related to rotation. This implies that normal old giant stars are not expected to be strong X-ray emitters. A possible explanation for the origin of the X-ray emission of these evolved stars could be if they are in synchronised binary systems, where the rotation period is equal to the orbital period, breaking thus the relation between X-ray emission and age. To test this hypothesis we calculated the X-ray colours expected for stars of different age and for binaries, in particular for RS CVn and BY Draconis. We found that observed Xray and infrared colours for dwarfs are compatible with young to intermediate age stars, while giant stars have colours compatible with RS CVn binaries, i.e. binaries where at least one of the stars is a giant star. The giant stars we find in our survey mostly have K spectral type, i.e. they are "yellow stars".

\section{Results}

We computed the $\log \mathrm{N}-\log \mathrm{S}$ curves in the soft band, i.e. the surface density of sources as a function of the sensitivity of our survey, for different bins of Galactic latitude. We find that the number of stars per square degree decreases with Galactic latitude. Although this result is not a surprise, it's the first time that is shown in $\mathrm{X}$-rays. We also see that the X-ray luminosity function has a varying slope with Galactic latitude. We compared our results with the expected curves from a modified version of the Galactic X-ray model from Guillout et al. 1996. At first glance we obtained compatible results, a varying slope of the X-ray luminosity function and a decreasing number of sources towards higher Galactic latitudes, reflecting the different scale-height of stars and the relative contribution of different populations. But for a given flux, the number of observed stars is higher than the predicted value. This result is not surprising since binaries have not been taken into account in our model. In other words, the excess of observed sources with respect to model predictions is due to the yellow giant stars that we have found in our survey, and are likely in binary systems.

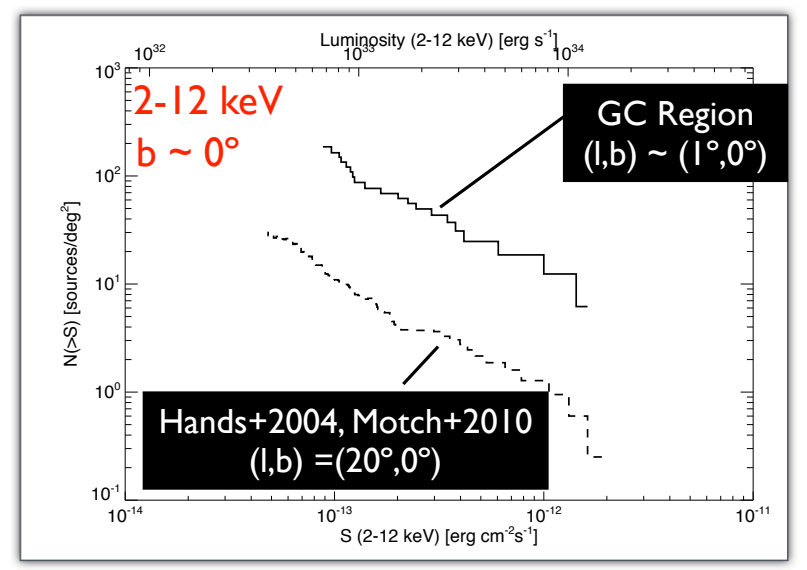

Figure 3: $\log \mathrm{N}-\log \mathrm{S}$ curves of hard sources $(>2 \mathrm{keV})$ in the direction of the Galactic Center. The expected extragalactic contribution from Mateos et al. 2008 has been subtracted.

We carried out the same exercise for hard sources, we constructed $\log \mathrm{N}-\log \mathrm{S}$ curves for sources harder than $2 \mathrm{keV}$. Since stars are not expected to contribute at this high energies, we subtracted the expected extragalactic contribution. The $\log \mathrm{N}-\log \mathrm{S}$ curves present an excess of sources with respect to the expected extragalactic contribution close to the Galactic Center region studied in this survey $\left(l \sim 0.9^{\circ}, b \sim 0^{\circ}\right)$. We compared this result with that obtained by Motch et al. 2010 at $l \sim 20^{\circ}, b \sim 0^{\circ}$ and obtained that the number of sources per square degree observed at a given flux decreases steeply with Galactic longitude in the direction of the Galactic Center. We obtained consistent results with Hong et al. 2009 based on Chandra observations of seven fields around the Galactic Center. This sources contribute to the Galactic Ridge emission, initially thought to be of diffuse origin. The nature of these sources is unknown, but if this population would be associated to the Galactic Center itself, the X-ray luminosities obtained are in the range $10^{33}-10^{34} \mathrm{erg} \mathrm{s}^{-1}$, i.e. values which are comparable with pre-HMXBs and pre-LMXBs. 


\section{Acknowledgement}

We would like to thank the organisers of this conference for their work and for giving us the opportunity to show our results.

\section{References}

[1] Anderson L. D., Bania T. M., Jackson J. M., Clemens D. P., Heyer M., Simon R., Shah R. Y., Rathborne J. M., 2009, ApJS, 181, 255 doi:10.1088/0067-0049/181/1/255

[2] Barcons, X., Carrera, F. J., Watson, M. G., et al. 2002, A\&A, 382, 522

[3] Barcons, X., Carrera, F. J., Ceballos, M. T., et al. 2007, A\&A, 476, 1191

[4] Della Ceca, R., Maccacaro, T., Caccianiga, A., et al. 2004, A\&A, 428, 383

[5] Favata, F., Sciortino, S., Rosner, R., \& Vaiana, G. S. 1988, ApJ, 324, 1010 doi:10.1086/165957

[6] Guillout, P., Haywood, M., Motch, C., \& Robin, A. C. $1996, A \& A, 316,89$

[7] Guillout, P., Sterzik, M. F., Schmitt, J. H. M. M., Motch, C., \& Neuhaeuser, R. 1998, A\&A, 337, 113

[8] Hands, A. D. P., Warwick, R. S., Watson, M. G., \& Helfand, D. J. 2004, MNRAS, 351, 31 doi:10.1111/j.1365-2966.2004.07777.x

[9] Hong, J. S., van den Berg, M., Grindlay, J. E., \& Laycock, S. 2009, ApJ, 706, 223 doi:10.1088/0004-637X/706/1/223

[10] López-Santiago, J., Micela, G., Sciortino, S., et al. 2007, A\&A, 463, 165

[11] Lutovinov A., Revnivtsev M., Gilfanov M., Shtykovskiy P., Molkov S., Sunyaev R., 2005, A\&A, 444, 821
[12] Mauerhan J. C., Muno M. P., Morris M. R., Stolovy S. R., Cotera A., 2010, ApJ, 710, 706

[13] Mateos, S., Warwick, R. S., Carrera, F. J., et al. 2008, A\&A, 492, 51

[14] Motch, C., Lopes de Oliveira, R., Negueruela, I., Haberl, F., \& Janot-Pacheco, E. 2007, in Astronomical Society of the Pacific Conference Series, Vol. 361, Active OB-Stars: Laboratories for Stellare and Circumstellar Physics, ed. A. T. Okazaki, S. P. Owocki, \& S. Stefl, 117

[15] Motch, C., Warwick, R., Cropper, M. S., et al. 2010, A\&A, 523, A92

[16] Nebot Gómez-Morán A. et al., 2013, A\&A, 553, A12

[17] Pfahl, E., Rappaport, S., \& Podsiadlowski, P. 2002, ApJ, 571, L37 doi:10.1086/341197

[18] Sazonov, S., Revnivtsev, M., Gilfanov, M., Churazov, E., \& Sunyaev, R. 2006, A\&A, 450, 117

[19] Sciortino, S., Favata, F., \& Micela, G. 1995, A\&A, 296,370

[20] Willems, B. \& Kolb, U. 2003, MNRAS, 343, 949

\section{DISCUSSION}

DAVID BUCKLEY: Can you give the percentages of the different optical counterpart types of the $\sim 300$ sources identified?

ADA NEBOT: Among the 316 classified sources there are three $\mathrm{T}$ Tauri stars, one Herbig Ae star, two cataclysmic variables, and four $\gamma$-Cas analogues. A few sources are extragalactic (in the highest Galactic latitude fields), and the remaining sources are stars, where about $10 \%$ are giants. Classification of giants was only done for stars with the best 2MASS photometry, meaning that the number of giants in our survey could be higher. 